\title{
Missed appointments among rifampicin-resistant tuberculosis (RR-TB) patients at a decentralised RR- TB outpatient clinic in Johannesburg, South Africa
}

\author{
R Gajee, ${ }^{1}$ MB ChB, MBA, MPH; K Schnippel, ${ }^{1,2}$ MPA; N Mthupha, ${ }^{1}$ BSc, MB ChB, Dip HIV Man; B Muzah, ${ }^{1}$ MB ChB, MSc, Dip HIV Man; \\ R H Berhanu, ${ }^{1,3}$ MD, DTM\&H, Dip HIV Man \\ ${ }^{1}$ Right to Care, Johannesburg, South Africa \\ ${ }^{2}$ Clinical HIV Research Unit, School of Clinical Medicine, Faculty of Health Sciences, University of the Witwatersrand, Johannesburg, South Africa \\ ${ }^{3}$ School of Medicine, University of North Carolina, Chapel Hill, NC, USA
}

Corresponding author: K Schnippel (kschnipp@gmail.com)

\begin{abstract}
Background. With the implementation of outpatient (ambulatory) decentralised rifampicin-resistant tuberculosis (RR-TB) treatment in South Africa (SA) since late 2011, the high rates of loss from treatment are a significant concern. Missed appointments lead to treatment interruptions and may contribute to amplification of resistance, ongoing transmission of RR-TB and an increased risk of morbidity and mortality to the patient.

Objective. To describe characteristics of patients who missed scheduled appointments during ambulatory RR-TB treatment.

Methods. The study was a retrospective, deidentified electronic medical record review of RR-TB patients at an outpatient clinic in Johannesburg, SA, from March 2013 to December 2014. Associations between missed appointments and clinical and demographic characteristics were analysed using time-to-event Cox proportional hazards regression.

Results. Of 172 patients who met the eligibility criteria, $53.5 \%$ missed at least one appointment and $39.5 \%$ missed three or more. More than half $(59.8 \%)$ of first missed appointments occurred within the first 3 months after treatment initiation. The median number of days from initiation until the first missed appointment was 82 (interquartile range 52 - 260.5). HIV-infected patients with a CD4 count of $\leq 100$ cells/ $\mu \mathrm{L}$ (adjusted hazard ratio (aHR) 4.25, 95\% confidence interval (CI) $1.49-12.18$ ), patients referred from an inpatient facility (aHR $1.96,95 \%$ CI 1.18 - 3.25) and patients aged 18 - 24 years as opposed to those aged 35 - 44 years (aHR 3.26, 95\% CI 1.20 - 8.84) were all more likely to miss one or more appointments.

Conclusion. HIV-infected patients with a low CD4 count, patients referred from inpatient care and young patients are at high risk of missing appointments and should receive interventions targeted at improving retention.
\end{abstract}

S Afr Med J 2016;106(9):912-917. DOI:10.7196/SAMJ.2016.v106i9.10570

Drug-resistant tuberculosis (DR-TB), especially TB resistant to rifampicin (RIF, RR-TB), threatens global TB control and is a major public health concern. In 2014, there were an estimated 480000 new cases of multidrug-resistant TB (MDR-TB) worldwide, and an estimated 190000 people died from MDR-TB. ${ }^{[1]}$ In 2014, South Africa (SA) initiated 11538 patients with RR-TB, including MDR$\mathrm{TB}$, on second-line TB treatment. Of the 2012 cohort of RR-TB cases, only $52 \%$ had a reported treatment outcome, the remainder having no outcome information reported. Among the patients with outcomes, the RR-TB treatment success rate was $49 \%$.

DR-TB cases are classified into the following categories, based on drug susceptibility testing: (i) monoresistance (resistance to one first-line anti-TB drug only); (ii) polydrug resistance (resistance to more than one first-line anti-TB drug, other than both isoniazid (INH) and RIF); (iii) MDR-TB (resistance to both INH and RIF); and (iv) extensive drug resistance (XDR-TB, resistance to any fluoroquinolone and at least one second-line injectable drug in addition to MDR-TB). RR-TB, defined as RIF resistance with or without resistance to other anti-TB drugs, includes monoresistant TB, MDR-TB and XDR-TB.

A review of cases reported to the national electronic register of drug-resistant TB (EDRweb) from 2009 to 2011 found that $29.5 \%$ of RR-TB patients were reported as lost from treatment. ${ }^{[2]}$ Other studies conducted in SA have reported rates of loss to follow-up in patients on treatment for MDR-TB of $20-30 \%{ }^{[3-5]}$ Associated risk factors include male gender, HIV co-infection, ${ }^{[3,5]}$ young age, previous RR-TB treatment, ${ }^{[5]}$ substance abuse and the attitude of healthcare workers. ${ }^{[6]}$

With the implementation of outpatient (ambulatory) decentralised RR-TB treatment in SA since late 2011, the potential for RR-TB patients to miss appointments is a concern. RR-TB patients who miss appointments may not have been able to visit the clinic because of TB morbidity or mortality or adverse drug events, but clinicians are not able to intervene if the patient is not in the clinic. Missed appointments also lead to treatment interruptions contributing to amplification of resistance, ongoing transmission of RR-TB, and an increased risk of morbidity and mortality. ${ }^{[7]}$

\section{Objective}

To describe the occurrence of missed appointments during decentralised outpatient RR-TB treatment, in order to inform strategies to reduce loss to follow-up, treatment failure and death.

\section{Methods}

\section{Study site and standard of care}

The study was conducted at a decentralised RR-TB outpatient clinic at Helen Joseph Hospital (HJH) in Johannesburg, SA.

With full-scale roll-out of GeneXpert MTB/RIF at the end of 2013, all individuals with presumptive TB in the SA public sector are also tested for RIF resistance. Those who are GeneXpert- 
negative but with symptoms indicative of TB may have additional smear microscopy or culture. Patients with a diagnosis of RR-TB are referred to the HJH RR-TB clinic on discharge from inpatient care or from other outpatient facilities for initiation of second-line TB treatment.

The SA National Department of Health has implemented a DR-TB management programme since early 2000 through the National TB Control Programme (NTCP). The NTCP guidelines up to 2011 stipulated that all MDR-TB patients should be hospitalised for 6 months or until sputum culture conversion (two consecutive negative cultures taken 1 month apart). The 2011 NTCP guidelines indicate that all smear microscopy-negative, culture-positive MDRTB patients should be started on ambulatory (outpatient) treatment; smear-positive MDR-TB patients should be hospitalised until smear conversion (two consecutive negative sputum smears); and nonambulatory MDR-TB and XDR-TB patients should be hospitalised until culture conversion. ${ }^{[8]}$

The NTCP treatment guidelines indicate that patients with RR-TB should be initiated on a standardised regimen for MDR-TB while further test results on drug resistance are pending. ${ }^{[9]}$ The standardised regimen has an intensive phase of 6 months, during which patients are treated with a second-line injectable (kanamycin or amikacin) and fluoroquinolone (moxifloxacin), plus terizidone, ethionamide and pyrazinamide. The continuation phase can vary in length, but should be at least 18 months following culture conversion and includes moxifloxacin, terizidone, ethionamide and pyrazinamide. Regimens can be individualised once there is evidence of either additional resistance (e.g. pre XDR-TB) or adverse reactions to the standardised regimen. Clinicians can add high-dose INH if there is an indication that it may still be an effective drug.

In SA, all HIV-infected TB patients, regardless of the level of TB drug resistance or CD4 count, are eligible for immediate initiation of antiretroviral (ARV) therapy ${ }^{[10]}$ HIV care is integrated with the RR-TB treatment; treatment and adverse event monitoring, including ARV pill collection, are provided for RR-TB and HIV co-infected patients at the HJH RR-TB clinic to reduce the number of clinic visits and improve adherence.

Patients enrolled for RR-TB management are given a scheduled appointment date to return to the HJH RR-TB clinic 2 weeks after RR-TB treatment initiation and then every 4 weeks (monthly) for 18 - 24 months. Patients can attend the clinic on any weekday during clinic hours at any point for an unscheduled appointment if they are feeling unwell. The patient can choose whether they want to attend the HJH RR-TB clinic or an alternative primary healthcare clinic closer to their home for daily directly observed treatment support and injections during the intensive phase. The treating clinician at the HJH RR-TB clinic retains responsibility for patient management throughout the 18 - 24 months of treatment.

\section{Study design}

The study was a retrospective, deidentified electronic medical record review. The site maintains electronic records of patients on a dedicated Microsoft Access database (2007 version, Microsoft, USA). For the study, a census of all patients enrolled in care at the site with documented RIF resistance between 1 March 2013 and 31 December 2014 was extracted. Follow-up data on visits to determine appointments missed and outcomes (treatment completed, died, lost from treatment) were extracted until the data extraction (28 February 2015), 18 months of treatment, death or loss to follow-up (defined as treatment interruption for 2 consecutive months ${ }^{[11]}$ ), whichever came earliest. Patients $<18$ years of age, those with INH monoresistant $\mathrm{TB}$ and those who were transferred to another treatment site were excluded from the analysis.
A missed appointment was defined as a monthly (4-weekly) appointment date scheduled by the clinic for which the patient did not have an actual appointment date recorded. The electronic medical record used at every patient visit has a data field where the next scheduled appointment date is captured, and it also has an adjacent data field where the actual appointment date on which the patient attended the clinic is captured. Scheduled appointment dates were set by the clinic staff during routine implementation and not through this analysis. Revised appointment dates to 'catch up' for previously delayed appointments or decisions as to whether an appointment was 3 weeks late or 1 week early after a missed appointment were made by the clinic staff as part of routine care.

\section{Statistical analysis}

Patients were categorised as having missed one or more appointments or no missed appointment. Associations between ever missing an appointment and clinical characteristics were analysed using timeto-event Cox proportional hazards regression. Clinical characteristics included HIV infection, CD4 count $\leq 100$ cells $/ \mu \mathrm{L}$ or $>100$ cells/ $\mu \mathrm{L}$ if HIV-infected, diagnosed at and referred from an outpatient or inpatient facility, any cough at RR-TB treatment initiation, pulmonary or extrapulmonary TB foci, resistance to RIF only or MDR-TB, and sputum smear microscopy-positive at initiation. Socioeconomic and demographic characteristics analysed included gender, age categories (18 - 24, 25 - 34, 35 - 44, 45 - 54 and $\geq 55$ years), employed or unemployed, SA or foreign national, primary, secondary or tertiary education, and living with children aged $\leq 5$ years. Hazard ratios adjusted for the other clinical and demographic characteristics (aHRs) were presented with $95 \%$ confidence intervals (CIs). Statistical significance was set at $p<0.05$. All analysis was done using Stata version 13.1 (StataCorp, USA).

\section{Ethics approval}

Ethics approval for the study was received from the Human Research Ethics Committee of the University of the Witwatersrand (Ref. no. M150341), and permission to conduct the research was obtained from $\mathrm{HJH}$. The study site routinely requests patients to provide informed consent for record review. Patients who declined or withdrew informed consent for review of medical records were excluded.

\section{Results}

\section{Patient characteristics}

During the study period, 172 patients who were initiated on RR-TB treatment at the study site from March 2013 to December 2014 met the eligibility criteria and were included in the analysis. The median age of patients initiated on RR-TB treatment was 36 years (interquartile range (IQR) 29 - 42), and 49.4\% were male (Table 1). Most patients (80.8\%) had a secondary school education, and $6.4 \%$ had a tertiary-level education. The majority of patients (83.7\%) were SA citizens, and $43.6 \%$ were employed.

HIV status was known for 169 patients (98.3\%); 148 (86.0\%) were HIV-infected, with a median CD4 count of 85 cells/ $\mu$ L (IQR 26 195). Of the HIV-infected patients, 90 (60.8\%) were on ART, with $61 / 148$ patients $(41.2 \%)$ having been on ART for $\geq 6$ months at initiation of RR-TB treatment.

Nearly half the patients ( $n=79,45.9 \%)$ were referred from inpatient care, i.e. they had recently been hospitalised for their condition. The majority of patients (90.1\%) had pulmonary TB. Most patients (70.3\%) were smear microscopy-negative or smear results were missing at initiation of second-line TB treatment. The median weight was $51 \mathrm{~kg}$ (IQR 45.1 - 59.3).

TB history was reported for 170 patients, with 103 (60.0\%) reporting no previous history of TB treatment, 53 (30.8\%) reporting a history of previous first-line TB treatment and 14 (8.1\%) reporting a history of receiving a TB regimen that included streptomycin. 


\section{Missed appointments and treatment outcomes}

The study found that $53.5 \%$ of patients $(n=92)$ missed at least one appointment; $39.5 \%(n=68)$ missed at least three

\begin{tabular}{lc} 
Table 1. Patient clinical and demographic characteristics at \\
initiation of second-line TB treatment $(\boldsymbol{N = 1 7 2})^{*}$ \\
\hline \begin{tabular}{ll} 
Description & $\boldsymbol{n}(\%)$ \\
\hline HIV status & $148(86.0)$ \\
$\quad$ Positive & $21(12.2)$ \\
$\quad$ Negative & \\
CD4 count $($ cells $/ \mu \mathrm{L})$ & $67(48.6)$ \\
$<101$ & $55(39.9)$
\end{tabular}
\end{tabular}

Antiretroviral status

On ART at RR-TB treatment initiation

$90(60.8)$

Not on ART at initiation

58 (39.2)

Antiretroviral history

Not on ART or on ART $<6$ mo at initiation

$69(53.1)$

On ART $\geq 6$ mo at initiation

$61(46.9)$

Referring site

Outpatient facility

$86(50.0)$

Inpatient facility

79 (45.9)

TB symptoms

Any cough at initiation

118 (68.6)

No cough or no report of cough

54 (31.4)

Site of TB

Pulmonary

$155(90.1)$

Extrapulmonary

17 (9.9)

Smear status

Microscopy positive

51 (29.7)

Microscopy negative or not reported

$121(70.3)$

Resistance

MDR-TB (resistant to INH and rifampicin)

38 (22.1)

RIF monoresistant TB

$82(47.7)$

$52(30.2)$

RIF resistant by GeneXpert MTB/RIF

103 (59.9)

No previous TB treatment reported

$53(30.8)$

Previous first-line TB treatment

$14(8.1)$

Previous retreatment TB regimen (streptomycin)

Weight at initiation $(\mathrm{kg})$

$$
<55
$$

$>54$

89 (51.7)

Continued ... appointments. Nineteen patients (11.0\%) died during the study period, $38(22.1 \%)$ were lost to follow-up, and $115(66.9 \%)$ had either completed treatment or remained on treatment at 18 months (Table 2). All 80 patients who had not missed an appointment had an outcome of either treatment completed or remaining on treatment at 18 months. Of the 92 patients who had missed one or more appointment, 19 (20.7\%) died, 38 (41.3\%) were lost from treatment and $35(38.0 \%)$ completed treatment or remained on treatment at 18 months.

\section{Time to missed appointments}

A total of 37940 person-days were analysed, and on average each patient contributed 221 days to the analysis. The time at risk of missed appointments was censored at 540 days (18 months). More than half $(59.8 \%)$ of all first missed appointments occurred in first 3 months of treatment (Fig. 1). The median number of days from

Table 1. (continued) Patient clinical and demographic characteristics at initiation of second-line TB treatment $(N=172)^{*}$

\begin{tabular}{ll}
\hline Description & $\boldsymbol{n}(\%)$ \\
\hline Gender & \\
Male & $85(49.4)$ \\
Female & $87(50.6)$
\end{tabular}

Age (years)

\section{$18-24$}

$25-34$

$35-44$

45 - 54

$\geq 55$

Education

Primary

Secondary 139 (80.8)

Tertiary

Citizenship

SA

144 (83.7)

Non-SA

Employment

Not employed or only day labour 95 (55.2)

Employed or self-employed

Children

Children aged $\leq 5$ years at home

$100(58.1)$

No children aged $\leq 5$ years at home

$64(37.2)$

Not reported

${ }^{*}$ Patients with missing data were excluded from the relevant analysis: HIV status $n=3,1.7 \%$; CD4 count if HIV-positive $n=16,11.6 \%$; referring site $n=7,4.1 \%$; TB history $n=2,1.2 \%$;
weight $n=9,5.2 \%$; employment status $n=2,1.2 \%$.

Table 2. Missed appointments and treatment outcome at 18 months or data extraction

\begin{tabular}{llll}
\hline Treatment outcome & $\begin{array}{l}\text { No missed appointments, } \\
\boldsymbol{n}(\%)\end{array}$ & $\begin{array}{l}\text { Missed } \geq \mathbf{1} \text { appointment/s, } \\
\boldsymbol{n}(\%)\end{array}$ & $\begin{array}{l}\text { Missed } \geq 3 \text { appointments, } \\
\boldsymbol{n}(\%)\end{array}$ \\
\hline On treatment or treatment completed & $80(100.0)$ & $35(38.0)$ & $21(30.9)$ \\
Died & $0(0)$ & $19(20.7)$ & $9(13.2)$ \\
Lost from treatment & $0(0)$ & $38(41.3)$ & $38(55.9)$ \\
Total & $80(46.5)$ & $92(53.5)$ & $68(39.5)$
\end{tabular}


treatment initiation until the first missed appointment was 82 days (IQR 52 - 260.5). The Kaplan-Meier curve demonstrated that missed appointments occurred earlier among patients who had an outcome of loss from treatment or death than among those who had an outcome of treatment completed or remaining on treatment at 18 months (Fig. 2). Missed appointments also occurred earlier after treatment initiation among patients referred from inpatient care than among those referred from outpatient facilities (Fig. 3).

\section{Predictors of missed appointments}

Adjusted Cox proportional hazards regression analysis demonstrated that HIV-infected patients with a CD4 count of $\leq 100$ cells/ $\mu \mathrm{L}$ were 4.25 times more likely to miss an appointment than those with a CD4 count of $>100$ cells $/ \mu \mathrm{L}$ (95\% CI 1.49 - 12.18) (Table 3). Patients aged 18 - 24 years were at a three times higher risk of missing an appointment compared with those aged 35 - 44 years (aHR 3.26, 95\% CI 1.20 - 8.84); patients aged 25 - 34 years were also at increased risk (aHR 1.94, 95\% CI 1.10 - 3.44). Patients who were referred from inpatient facilities were twice as likely to miss an appointment as those who were referred from outpatient facilities (aHR 1.96, 95\% CI 1.18 - 3.25). When the site of TB was pulmonary, patients were almost three times more likely to miss an appointment compared with patients who had an extrapulmonary site of TB (aHR 2.77, 95\% CI 1.04 - 7.34). Although not statistically significant, patients with a tertiary-level education were less likely to miss an appointment than patients with a primary school education (aHR $0.28,95 \%$ CI $0.07-1.07)$.

\section{Discussion}

The finding that $53.5 \%$ of patients missed one or more appointments during outpatient treatment for RR-TB is of concern. Patients referred from inpatient care, HIV-infected patients with a CD4 count $\leq 100$ cells $/ \mu \mathrm{L}$ and young patients (18 - 24 years) are at a significantly increased risk of missing an appointment. Missing at least one scheduled appointment at the RR-TB clinic was strongly associated with poor treatment outcomes; patients missing three or more appointments were even less likely to complete treatment successfully.

The proportion of patients missing at least one appointment was higher than was found in a study on missed appointments during the first 6 months among HIV-infected patients attending an ART outpatient clinic at the same facility ${ }^{[12]}$ (35\% of 4476 patients

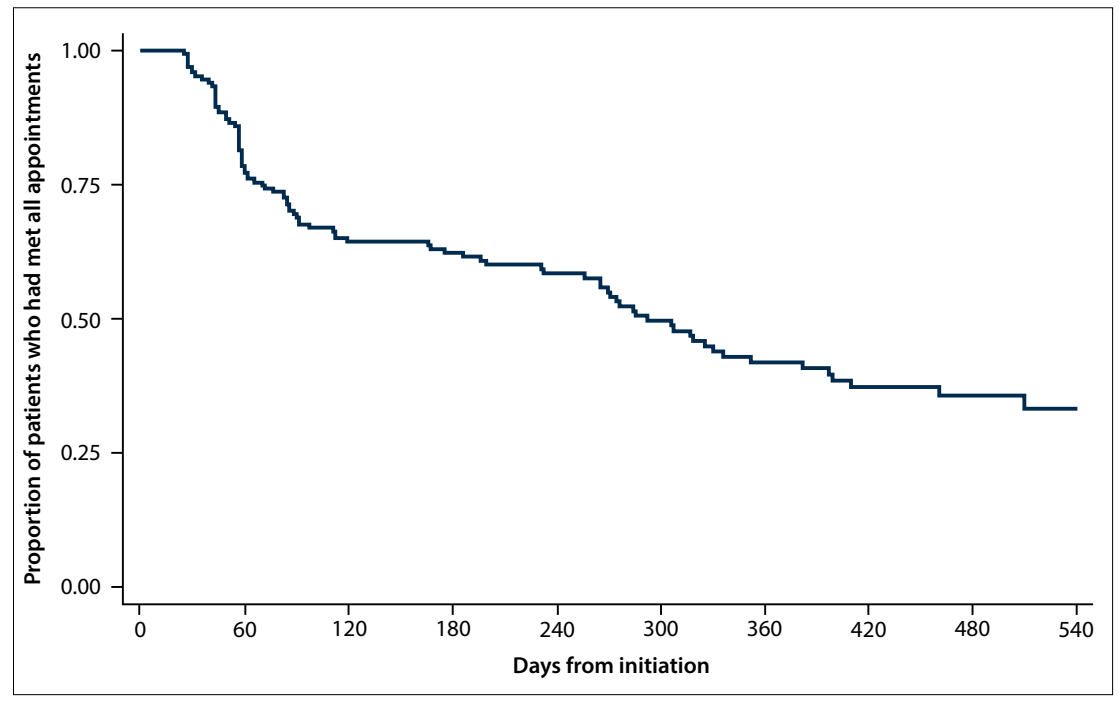

Fig. 1. Kaplan-Meier survival function: time from initiation of RR-TB treatment until first monthly appointment missed.

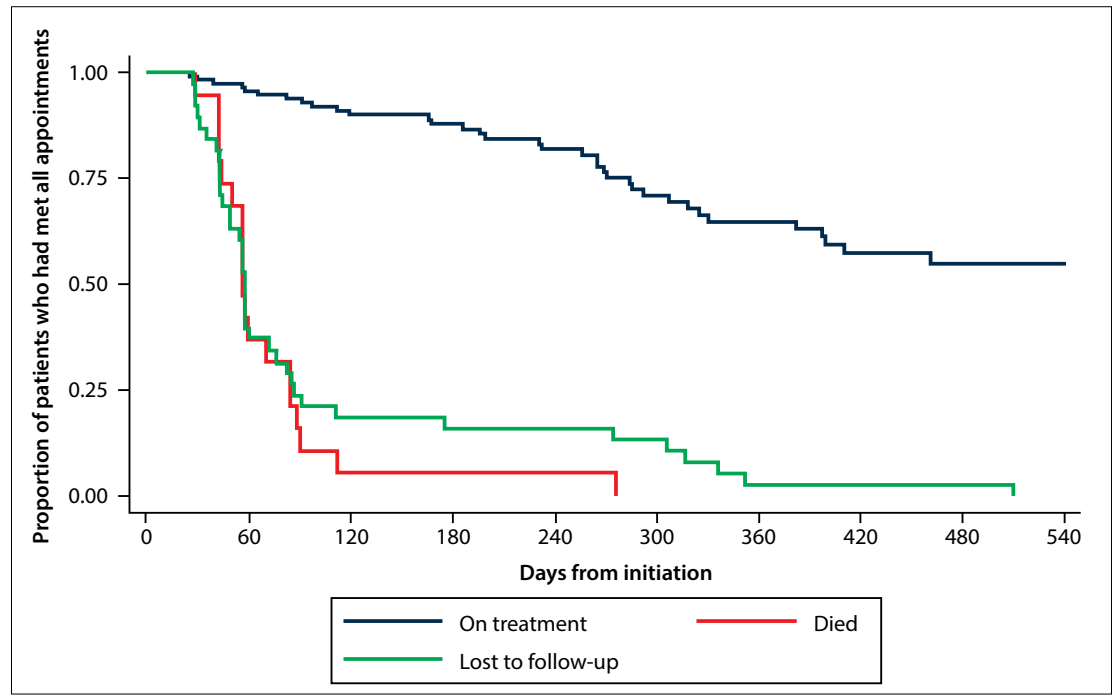

Fig. 2. Kaplan-Meier survival function: time from treatment initiation until first missed appointment by treatment status at censoring.

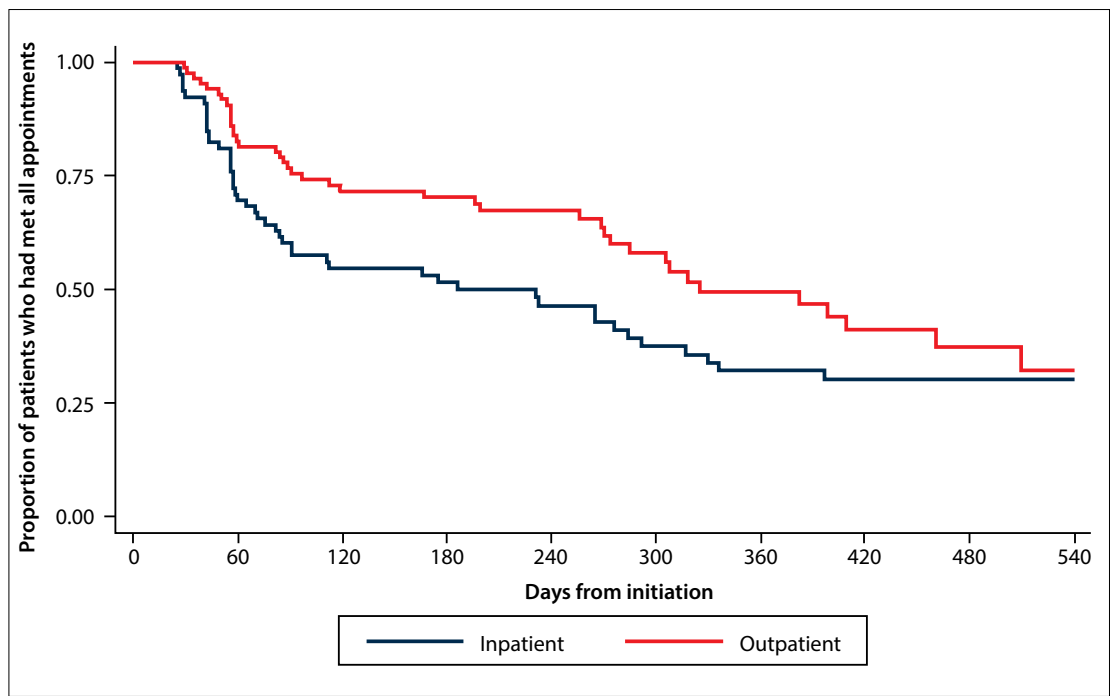

Fig. 3. Kaplan-Meier survival function: time from initiation of RR-TB treatment until first monthly appointment missed, by referring site type (outpatient or inpatient facility). 
Table 3. Cox proportional hazards, risk of missing one or more appointments, time-toevent regression

\begin{tabular}{|c|c|c|}
\hline Description & $\begin{array}{l}\text { Unadjusted HR } \\
(95 \% \text { CI })\end{array}$ & $\begin{array}{l}\text { Adjusted HR* } \\
(95 \% \text { CI })\end{array}$ \\
\hline \multicolumn{3}{|l|}{ HIV status } \\
\hline Negative & Reference & Reference \\
\hline Positive, $\mathrm{CD} 4>100$ cells $/ \mu \mathrm{L}$ & $1.37(0.66-2.82)$ & $2.94(1.07-8.10)$ \\
\hline Positive, $\mathrm{CD} 4<101$ cells $/ \mu \mathrm{L}$ & $1.95(0.95-4.01)$ & $4.25(1.49-12.18)$ \\
\hline \multicolumn{3}{|l|}{ Referring site } \\
\hline Inpatient & $1.53(1.01-2.32)$ & $1.96(1.18-3.25)$ \\
\hline \multicolumn{3}{|l|}{ TB symptoms } \\
\hline Any cough & $0.84(0.54-1.29)$ & $0.72(0.43-1.21)$ \\
\hline \multicolumn{3}{|l|}{ Site of TB } \\
\hline Pulmonary TB & $1.68(0.78-3.64)$ & $2.77(1.04-7.34)$ \\
\hline \multicolumn{3}{|l|}{ Resistance } \\
\hline MDR-TB & $0.77(0.46-1.28)$ & $0.83(0.44-1.58)$ \\
\hline \multicolumn{3}{|l|}{ TB history } \\
\hline No prior TB & $0.96(0.62-1.48)$ & $1.25(0.74-2.11)$ \\
\hline \multicolumn{3}{|l|}{ Weight } \\
\hline$<55 \mathrm{~kg}$ & $0.92(0.60-1.41)$ & $0.61(0.36-1.02)$ \\
\hline \multicolumn{3}{|l|}{ Smear microscopy } \\
\hline Positive & $0.83(0.53-1.30)$ & $1.10(0.62-1.94)$ \\
\hline \multicolumn{3}{|l|}{ Gender } \\
\hline Male & $0.84(0.56-1.27)$ & $0.82(0.48-1.40)$ \\
\hline \multicolumn{3}{|l|}{ Age (years) } \\
\hline $18-24$ & $1.41(0.65-3.07)$ & $3.26(1.20-8.84)$ \\
\hline $25-34$ & $1.93(1.20-3.12)$ & $1.94(1.10-3.44)$ \\
\hline $35-44$ & Reference & Reference \\
\hline $45-54$ & $1.11(0.58-2.12)$ & $1.36(0.63-2.93)$ \\
\hline$\geq 55$ & $0.66(0.20-2.16)$ & $1.22(0.33-4.51)$ \\
\hline \multicolumn{3}{|l|}{ Education } \\
\hline Primary & Reference & Reference \\
\hline Secondary & $0.77(0.43-1.36)$ & $0.59(0.31-1.15)$ \\
\hline Tertiary & $0.29(0.08-0.99)$ & $0.28(0.07-1.07)$ \\
\hline \multicolumn{3}{|l|}{ Citizenship } \\
\hline Non-SA & $1.25(0.75-2.09)$ & $1.04(0.52-2.06)$ \\
\hline \multicolumn{3}{|l|}{ Employment } \\
\hline Not employed & $1.11(0.73-1.68)$ & $0.75(0.45-1.27)$ \\
\hline \multicolumn{3}{|l|}{ Children } \\
\hline Children $\leq 5$ years & $0.86(0.56-1.32)$ & $1.03(0.63-1.71)$ \\
\hline
\end{tabular}

missed one or more appointment). Conversely, the rates were conservative compared with a more detailed analysis of missed treatment doses (treatment interruptions) during RR-TB treatment at a site in the Philippines, ${ }^{[7]}$ where $93 \%$ of patients had at least one documented treatment interruption. The studies have in common that missing appointments was associated with poor outcomes.

This study was a retrospective review of medical records and therefore cannot determine either the reasons for the missed appointments or the direction of causality. The 'treatment journey' for patients on RR-TB treatment, especially those co-infected with HIV, can be a challenge for patients, and reasons for the treatment interruptions can be related to the patient or the healthcare system. ${ }^{[13]}$ The proportion of RR-TB patients co-infected with HIV (86\%) was much higher than the average for drug-susceptible TB patients in SA (61\%). ${ }^{[1]}$ The study also found a high degree of immunosuppression among RR-TB patients co-infected with HIV (median CD4 count 85 cells $/ \mu \mathrm{L}$, IQR 26 - 195). This is of concern, as low CD4 counts are a strong predictor of opportunistic infections and mortality among HIV-infected patients. Furthermore, the finding that a high proportion of HIV-infected patients (39.2\%) were not on ART at the time of RR-TB treatment initiation confirms that they were not initiated on effective treatment for their HIV. The high risk of missed appointments among HIV-positive patients with a CD4 count of $\leq 100$ cells/ $\mu \mathrm{L}$ (aHR 4.25, 95\% CI 1.49 - 12.18) and patients referred from inpatient care reported here (aHR 1.96, 95\% CI 1.18 - 3.25) may be a result of these patients being too sick to attend outpatient appointments, and loss from treatment may include unreported deaths. ${ }^{[14]}$ The missed appointments may also be a result of the inpatients not having a previous connection to the clinic providing daily treatment; a study from the same site found that $23 \%$ of drugsensitive TB patients diagnosed at the hospital never linked to the primary healthcare clinic for TB treatment. ${ }^{[15]}$ Overall, 59.8\% of missed appointments occurred within the first 3 months after RR-TB treatment initiation. Missed appointments occurred earlier among patients referred from inpatient care than among those referred from outpatient care, again suggesting that sicker patients are more prone to missed appointments. As has been found with $\mathrm{ART},{ }^{[14]}$ provider- and patientcentred support to reduce early loss from treatment should be targeted at the first months after initiation.

Our finding that patients aged 18 - 24 years were at a three times higher risk and patients aged 25 - 34 years at a two times higher risk of missing an appointment compared with patients aged 35 - 44 years (aHR 3.26, 95\% CI 1.20 - 8.84) was similar to the findings of other studies investigating loss to follow-up among DR-TB patients in decentralised care. A decentralised DR-TB site in Khayelitsha, Western Cape Province, SA, found that patients aged $13-25$ years were at a three times increased risk of loss to follow-up (OR 3.0, 95\% CI $1.1-8.1) .{ }^{[4]}$ It is critical to develop targeted and age-appropriate interventions for the young patient initiated on RR-TB treatment to reduce missed appointments and loss to follow-up. 


\section{Study limitations}

The study was limited to one site, so the results on missed appointments may not be generalisable to all outpatient RR-TB clinics. The study was retrospective and therefore limited to the data that were available. There was no information on patients who transferred out to other sites after their transfer. Despite these limitations, this study adds to the body of literature describing important demographic and clinical characteristics associated with missing appointments and loss to follow-up during the delivery of outpatient RR-TB treatment.

\section{Conclusion}

There are high rates of missed appointments among RR-TB patients in decentralised outpatient care. In order to achieve global targets for RR-TB treatment success, retention in treatment must be improved. The findings of this study highlight which patients need additional support in order to remain in care and prevent further transmission, morbidity and mortality. These interventions should look at both provider and patient reasons for loss to treatment, and may include patient reminders, tracing in the community, hospitalisation for patients in a poor clinical condition, dedicated patient transport, or other solutions.

1. World Health Organization. Global Tuberculosis Report 2014. Geneva: WHO, 2014. www.who.int/tb/ publications/global_report/en/ (accessed 15 July 2015).

2. Schnippel K, Shearer K, Evans D, et al. Predictors of mortality and treatment success during treatment for rifampicin-resistant tuberculosis within the South African National TB Programme, 2009 to 2011: A cohort analysis of the national case register. Int J Infect Dis 2015;39(2015):89-94. DOI:10.1016/j.ijid.2015.09.002

3. Brust JC, Gandhi NR, Carrara H, Osburn G, Padayatchi N. High treatment failure and default rates for patients with multidrug-resistant tuberculosis in KwaZulu-Natal, South Africa, 2000-2003. Int J Tuberc Lung Dis 2010;14(4):413-419.
4. Farley JE, Ram M, Pan W, et al. Outcomes of multi-drug resistant tuberculosis (MDR-TB) among a cohort of South African patients with high HIV prevalence. PLoS One 2011;6(7):e20436. DOI:10.1371/ journal.pone. 0020436

5. Cox H, Hughes J, Daniels J, et al. Community-based treatment of drug-resistant tuberculosis in Khayelitsha, South Africa. Int J Tuberc Lung Dis 2014;18(4):441-448. DOI:10.5588/ijtld.13.0742

6. Finlay A, Lancaster J, Holtz TH, et al. Patient- and provider-level risk factors associated with default from tuberculosis treatment, South Africa, 2002: A case-control study. BMC Public Health 2012;12:56. DOI:10.1186/1471-2458-12-56

7. Podewils LJ, Gler MTS, Quelapio MI, Chen MP. Patterns of treatment interruption among patients with multidrug-resistant $\mathrm{TB}$ (MDR TB) and association with interim and final treatment outcomes. PLoS One 2013;8(7):e70064. DOI:10.1371/journal.pone.0070064

8. Directorate: Drug-Resistant Tuberculosis, TB \& HIV, Chief Directorate: TB Control and Management. Multi-drug Resistant Tuberculosis: A Policy Framework on Decentralised and Deinstitutionalised Management for South Africa. Pretoria: South African National Department of Health, 2011. http://www.tbfacts.org/wp-content/uploads/2015/08/SA-MDR-TB-Policy.pdf (accessed 29 July 2016).

9. Directorate: Drug-Resistant Tuberculosis, TB \& HIV. Management of Drug-Resistant Tuberculosis: Policy Guidelines. Pretoria: South African National Department of Health, 2011. Tuberculosis: Policy Guidelines. Pretoria: South African National Department of Health, 2011.
http://www.hst.org.za/publications/management-drug-resistant-tuberculosis-policy-guidelines (accessed 15 July 2015)

10. South African National Department of Health. The South African Antiretroviral Treatment Gouth African National Department of Health. The South African Antiretroviral Treatment
Guidelines. Pretoria: NDoH, 2013. http://www.sahivsoc.org/upload/documents/2013\%20ART\%20 Guidelines-Short\%20Combined\%20FINAL\%20draft\%20guidelines\%2014\%20March\%202013.pdf (accessed 15 July 2015).

11. World Health Organization. Definitions and reporting framework for tuberculosis - 2013 revision. 2013. http://apps.who.int/iris/handle/10665/79199 (accessed 15 July 2015).

12. Brennan AT, Maskew M, Sanne I, Fox MP. The importance of clinic attendance in the first six months on antiretroviral treatment: A retrospective analysis at a large public sector HIV clinic in South Africa. J Int AIDS Soc 2010;13:49. DOI:10.1186/1758-2652-13-49

13. Loveday M, Padayatchi N, Voce A, Brust J, Wallengren K. The treatment journey of a patient with multidrug-resistant tuberculosis in South Africa: Is it patient-centred? Int J Tuberc Lung Dis 2013;17(10 Suppl 1):56-9. DOI:10.5588/ijtld.13.0101

14. Fox MP, Brennan A, Maskew M, MacPhail P, Sanne I. Using vital registration data to update mortality among patients lost to follow-up from ART programmes: Evidence from the Themba mortality among patients lost to follow-up from ART programmes: Evidence from the Themba
Lethu Clinic, South Africa. Trop Med Int Health 2010;15(4):405-413. DOI:10.1111/j.13653156.2010.02473.

5. Voss de Lima Y, Evans D, Page-Shipp L, et al. Linkage to care and treatment for TB and HIV among people newly diagnosed with TB or HIV-associated TB at a large, inner city South African hospital. PLoS One 2013;8:e49140. DOI:10.1371/journal.pone.0049140

Accepted 12 July 2016. 